\title{
Use of Severity Indicators in a Public Health Surveillance System
}

\author{
Rhonda A. Lizewski*1, Howard Burkom², Joseph Lombardo², Christopher Cuellar², \\ Yevgeniy Elbert ${ }^{2}$ and Julie Pavlin ${ }^{1}$
}

${ }^{1}$ Armed Forces Health Surveillance Center, Silver Spring, MD, USA; ${ }^{2}$ Johns Hopkins Applied Physics Lab, Laurel, MD, USA

\section{Objective}

To evaluate new severity indicators that mimic a public health professional or clinician's judgment in determining the severity of a public health event when detected by a surveillance system.

\section{Introduction}

Data streams related to case severity have been added to the Electronic Surveillance System for the Early Notification of Community-based Epidemics (ESSENCE), a disease-monitoring application used by the Department of Defense (DoD), as an additional analytic capability to alert the user when indications for events requiring expanded medical resources exist in clinical data streams. Commonly used indicators are admission and death, but fatalities are rare and many DoD clinics lack admitting capability, so we sought to derive additional severity indicators from outpatient records. This abstract describes the technical details and the thought process behind two novel derived indicators: Sick-in-Quarters (SIQ) and Escalating Care.

\section{Methods}

We analyzed 3.75 years of ESSENCE outpatient records from over 400 Military Treatment Facilities (MTFs) with regard to appointment type, disposition, "bounce-backs" and Evaluation and Management (E/M) codes. The appointment type field contains codes such as "acute," "routine," and "established." Visits designated as "acute" may require more immediate attention, but usage of this code varies widely among MTFs. For patients not admitted, the most common dispositions are released without limitations and SIQ. SIQ is a unique disposition which directs military personnel not well enough to return to duty to remain in their quarters for a specified length of time. A "bounce-back" refers to a patient visit at an Emergency Department (ED) within 24 to 72 hours of an earlier visit with similar symptoms. These patients require more attention and careful consideration in diagnosis and treatment. Many MTFs do not have EDs, so we defined the term "Escalating Care" to include bounce-backs and also similar-presenting return visits to primary care clinics within 72 hours, but excluding returns with "ESTABLISHED" or "ROUTINE" Appointment Types or follow-up indication in the chief complaint. The resulting severity indicator was daily counts of these Escalating Care visits. In outpatient records, E/M codes are 5-digit strings, used for billing purposes, whose definitions can indicate presentations requiring extended care. We tabulated E/M codes for all MTFs to determine codes most indicative of severe cases in practice. Odds ratios (ORs) were applied for this purpose to individual code usage during and outside outbreak intervals. Correlation analysis also helped to select and confirm the set of severity indicators.

\section{Results}

Using data from 36 ILI health events with severe patient outcomes, respective ORs of the E/M, SIQ, and Escalating Care indicators were 6.9, 7.0, and 5.5. Analogous ORs for 16 GI events were 13.3, 12.3, and 11.7. Figure 1 shows daily counts of ILI-related visits for a sample MTF and associated counts of Acute appointment types and SIQ dispositions, typical of many MTFs where Acute appointment types are coded liberally but SIQ peaks are more likely to indicate need for extended care.

\section{Conclusions}

Several indicators of likely severe outcomes have been derived from DoD ESSENCE outpatient data. The novel SIQ and Escalating Care indicators are combined with a weighted $\mathrm{E} / \mathrm{M}$ indicator and the rarer admissions/deaths to identify events requiring expanded medical resources. Correlation and OR analysis verified that medical record field usage varies in practice among facilities and may differ from published intent. A combination of medical and coding expertise with historical data analysis was required to derive reliable indicators.

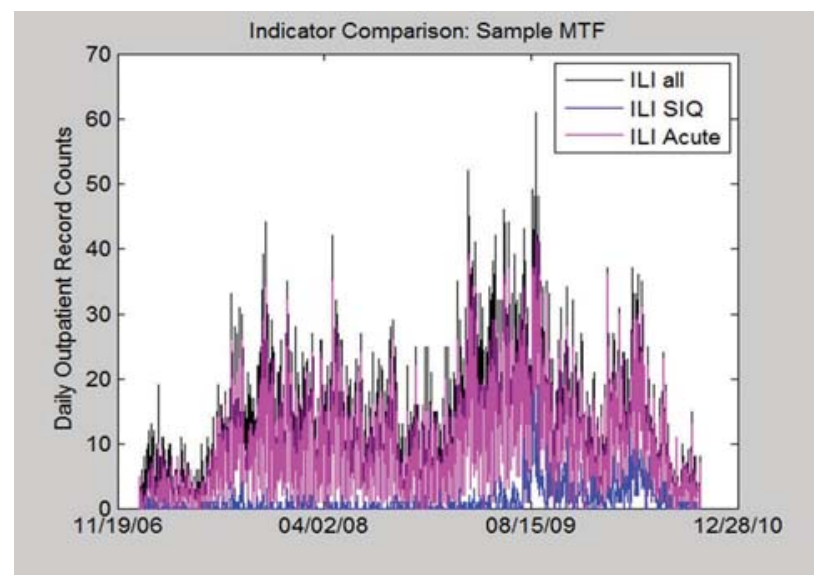

Figure 1: Daily counts of ILI-related visits and associated Acute appointment types and SIQ disposition counts from a sample facility.

\section{Keywords}

Severity; ESSENCE; SIQ; Escalating care

\section{*Rhonda A. Lizewski}

E-mail: rhonda.a.lizewski.mil@mail.mil 